\title{
BMJ Open Health professionals' experiences of tuberculosis cohort audit in the North West of England: a qualitative study
}

\author{
Selina K Wallis, ${ }^{1}$ Kate Jehan, ${ }^{2}$ Mark Woodhead, ${ }^{3}$ Paul Cleary, ${ }^{4}$ Katie Dee, ${ }^{4}$ \\ Stacey Farrow, ${ }^{5}$ Paddy McMaster, ${ }^{6}$ Carolyn Wake, ${ }^{4}$ Jenny Walker, ${ }^{7}$ D J Sloan,,${ }^{1,8}$ \\ S B Squire ${ }^{1,9}$
}

To cite: Wallis SK, Jehan $\mathrm{K}$, Woodhead M, et al. Health professionals' experiences of tuberculosis cohort audit in the North West of England: a qualitative study. BMJ Open 2016;6:e10536.

doi:10.1136/bmjopen-2015010536

- Prepublication history and additional material is available. To view please visit the journal (http://dx.doi.org/ 10.1136/bmjopen-2015010536).

Received 12 November 2015 Revised 16 February 2016 Accepted 16 February 2016

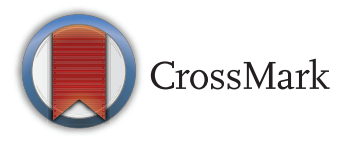

For numbered affiliations see end of article.

Correspondence to Selina K Wallis; selinawallis@hotmail.com and Bertie Squire; Bertie.Squire@Istmed.ac.uk

\section{ABSTRACT}

Objectives: Tuberculosis cohort audit (TBCA) was introduced across the North West (NW) of England in 2012 as an ongoing, multidisciplinary, systematic case review process, designed to improve clinical and public health practice. TBCA has not previously been introduced across such a large and socioeconomically diverse area in England, nor has it undergone formal, qualitative evaluation. This study explored health professionals' experiences of the process after 1515 cases had been reviewed.

Design: Qualitative study using semistructured interviews. Respondents were purposively sampled from 3 groups involved in the NW TBCA: (1) TB nurse specialists, (2) consultant physicians and (3) public health practitioners. Data from the 26 respondents were triangulated with further interviews with key informants from the TBCA Steering Group and through observation of TBCA meetings.

Analysis: Interview transcripts were analysed thematically using the framework approach.

Results: Participants described the evolution of a valuable 'community of practice' where interprofessional exchange of experience and ideas has led to enhanced mutual respect between different roles and a shared sense of purpose. This multidisciplinary, regional approach to TB cohort audit has promoted local and regional team working, exchange of good practices and local initiatives to improve care. There is strong ownership of the process from public health professionals, nurses and clinicians; all groups want it to continue. TBCA is regarded as a tool for quality improvement that improves patient safety.

Conclusions: TBCA provides peer support and learning for management of a relatively rare, but important infectious disease through discussion in a no-blame atmosphere. It is seen as an effective quality improvement strategy which enhances TB care, control and patient safety. Continuing success will require increased engagement of consultant physicians and public health practitioners, a secure and ongoing funding stream and establishment of clear reporting mechanisms within the public health system.

\section{Strengths and limitations of this study}

- This study is the first to formally evaluate the perceptions of participants at tuberculosis cohort audit (TBCA).

- Our qualitative data set included a large and extensive number of stakeholders from three main cadres that participate in TBCA.

- The unique geographic configuration and implementation of the North West TBCA may mean that the findings are not easily transferred to other areas without careful adaptation.

\section{INTRODUCTION}

Growing pressure on the National Health Service (NHS) to provide safe effective healthcare within a limited budget ${ }^{1}$ has focused attention on ways to promote efficient working of diverse multidisciplinary groups.

Where infectious diseases such as tuberculosis (TB) are rare, ${ }^{2}$ issues can arise where public health funds may be diverted to other purposes, and clinicians may lack diagnostic skills or knowledge of current treatment options. ${ }^{3}$ Countries with a low incidence of TB need to ensure they have continuing and adequate vigilance systems to detect and treat $\mathrm{TB}$ cases and thereby prevent transmission. ${ }^{4}$

TB predominantly affects vulnerable and hard to reach groups and requires the coordination of multiple sectors and organisations for effective treatment, control and prevention. ${ }^{4}$

TB cohort audit (TBCA; often called TB cohort review) was introduced across the North West (NW) of the UK in 2012. TBCA has not previously been introduced across such a large and socioeconomically diverse 
area in the UK, nor has it undergone formal, qualitative evaluation. There is increasing interest in TBCA as a means of improving patient safety, disseminating knowledge, facilitating the implementation of evidence-based practice and improving staff morale. ${ }^{56}$

In order for health services to be effective and translate into meaningful patient care outcomes, it is necessary to evaluate not only summative but also formative outcomes to assess if, how and why implementation is effective in a specific setting. Individual beliefs govern behaviour change and ultimately determine whether there is engagement with change initiatives. ${ }^{7}$

This paper aims to build a greater understanding of how TBCA in the NW was perceived by groups of differing health professionals, across a wide geographic area, dealing with a low incidence but high consequence disease. Our findings will be of relevance to those interested in establishing and supporting effective TBCA and in understanding some of the factors that contribute to successful communities of practice in healthcare.

\section{BACKGROUND}

TBCA was first developed in Tanzania in the 1990s and rapidly became an integral part of the international TB control effort. ${ }^{8}$ It was subsequently adapted for use in New York during the 1990s, where it contributed to an increase in completion rates and a reduction in $\mathrm{TB}$ and multidrug-resistant TB cases. $^{9}$ TBCA was first implemented in the UK in North Central London (NCL) in 2010, and has been recommended in the National Institute for Health and Care Excellence (NICE) guidance since 2012. ${ }^{10}$ Evaluation of the London model of TBCA, where each area has a separate process, found improvements in case management, contact tracing and identification of service issues, but suggested that TBCA should be monitored after implementation. ${ }^{11}$

The UK has one of the highest incidence rates of TB in Western Europe, with particularly high rates in metropolitan areas and linked to deprivation. ${ }^{12}{ }^{13}$ In the year of this study (2014), London Public Health England (PHE) Centre and West Midlands PHE Centre reported the highest TB notification rates in the UK (30.1 per 100000 and 13.7 per 100000$).{ }^{14}$ Both cities run focused, urban, TBCA processes. The average rate across the NW was low at 9.1 per 100000 but urban Manchester's notification rate was 31.3 per 100000 (3-year average annual rate 2012-2014). ${ }^{14}$

Owing to these pockets of high incidence of TB in the NW, TBCA was implemented (in line with international and national best practice ${ }^{810} 15$ ) in 2012 as a single, coordinated process across the whole range of rural, urban and socioeconomic contexts of the region. ${ }^{16}$ Objectives of the NW TBCA relate to case management and TB control but also include provision of ongoing training and education for staff and provision of a forum for open discussion. ${ }^{17}$

\section{STUDY DESIGN}

\section{Context of the healthcare system}

TB care in the UK is coordinated by lead TB consultant physicians who work within secondary care hospital and community trusts which are part of the NHS. This is a system of universal free healthcare funded through taxation. Once TB is confirmed, TB treatment is initiated either in the community or in hospital, depending on the clinical severity or complexity of each case. Once stabilised, patients are supported in the community by TB nurse specialists. Each case undergoes a standardised risk assessment and direct observation of therapy is reserved for the most complex cases.

\section{TBCA process and costs}

As with other models of TBCA, the process engages key stakeholders in TB care and control (nurses, doctors and public health staff). The budget lines of TBCA in the model described here have been identified, but not yet formally costed. They include the salary of an $80 \%$ full time equivalent (FTE) coordinator, the hire of meeting venues, the purchase of refreshments and the travel costs of TBCA Chairs (see below). There are also travel costs for the meetings of the TBCA Steering Group which established, and continues to monitor and develop the system of quarterly multidisciplinary meetings in four operational footprints: Cheshire and Merseyside, Cumbria and Lancashire, North Manchester and South Manchester, each covering both urban and rural contexts and a number of NHS Trusts. The time devoted to preparation of cases and participants' time in meetings are regarded as part of service delivery and quality assurance.

Cases are identified by the TBCA coordinator from Enhanced Tuberculosis Surveillance (ETS) notifications during a 3-month period ending 6 months before each TBCA meeting. TB nurse specialists complete a bespoke, anonymised electronic data collection form for each case and present the completed forms at each meeting.

TBCA meetings are chaired by clinicians (some now co-chaired by TB nurse specialists) from outside the footprint represented in a given meeting. The chairperson opens the meeting with introductory slides outlining results of programme performance from all four operational footprints against 10 pre-specified outcome measures. $^{18} \mathrm{~TB}$ nurses then present the cases. During and after each case, key data are checked and corrected. Challenges in case management, service strengths, weaknesses and staff training needs emerge from the discussion that surrounds each case and are flagged for action through the steering group by the Chair. Data are collated live by Public Health England staff and results on outcome measures are fed back at the end of each meeting. ${ }^{14}$ Thus, TBCA differs from conventional multidisciplinary case conferences in that all cases are discussed retrospectively with a public health focus on a pre-specified set of outcome measures rather than a 
focus on solving individual case management problems for selected, difficult cases.

TB nurse specialists are key to the process; across the NW, they have a wide variety of employers and contract types ranging from full time to part time. For some, only a small percentage of the workload is devoted to $\mathrm{TB}$.

\section{Study approach and data collection}

TBCA is considered a complex intervention and it is recognised that evaluations of this type of initiative should include a qualitative design to enable in-depth exploration of participants' experiences from their own perspectives. ${ }^{19}$ The aim of this study was therefore to qualitatively explore and understand perceptions and experiences of TBCA with three main stakeholder groups: TB nurse specialists, consultant physicians and public health practitioners. The epistemology underpinning the study is interpretivist, understanding knowledge as subjective and prioritising the individual meanings participants assign to their own experiences, knowledge that is inaccessible through experimental methods. $^{20} 21$ Face-to-face, semistructured interviews were considered the most suitable primary data collection tool to access this knowledge and to enable flexible, in-depth exploration of the issues. ${ }^{22}$ Interviews were conducted by SW, a public health researcher trained in qualitative research, independent of TBCA. These interviews were supplemented with additional key informant interviews with members of the NW TBCA Steering Group.

Interviews for this study took place in January to March 2014 (approximately 2 years after TBCA was implemented in the region and after 1515 TB cases had been reviewed). Topic guides were developed for the interviews, based on discussion with the cohort audit coordinator, steering group and a review of the existing literature. These were piloted and refined in an iterative process throughout the study. Interviews were further triangulated by observation undertaken by SW during TBCA meetings. ${ }^{23}$

The study is presented in line with the Consolidated Criteria for Reporting Qualitative Research (COREQ) ${ }^{24}$ (see online supplement file A).

\section{Sampling and participant recruitment}

Maximum variation purposive sampling was used to select potential staff participants and gain perspectives from a broad range of representative healthcare professionals. ${ }^{25}$ Sampling was intended to include gender balance; professionals working in high and low incidence areas; mix of professional roles and working patterns; mix of regular and less regular attendance at cohort. On the contact list for the TBCA, there are approximately 125 clinicians (92 males/33 females), 49 nurses (3 males/46 females), 43 other-PHE leads, epidemiologists, local authority (LA) leads, public health (PH) leads, Admin other TB leads (12 males/31 females).
The larger share of female nurses and male consultants in the sample reflects recruitment patterns to these professions nationally. ${ }^{26}$ Twenty-eight participants were originally selected from the three groups across the four geographic 'footprints' and contacted by the researcher by email. Twenty-six individuals were interviewed (one participant declined to take part and one was unable to schedule time to be interviewed) in private rooms chosen by the participants. Numbers of participants interviewed within the professional groups represented the proportions in which they attend TBCA. Duration of the interviews was approximately $1 \mathrm{~h}$, audio recorded and supplemented with notes taken by the researcher.

Key informants were individuals with a strategic in-depth knowledge of $\mathrm{TBCA}^{27}$ who were instrumental in running of TBCA-(Chairs, epidemiologists/PHE TB leads, steering group members) and were purposively sampled from across the four geographic subfootprints. Eight participants were selected, two from each footprint.

\section{Ethical procedure}

No risks to interviewees were anticipated from participation in the study. We obtained informed consent prior to the start of the interviews, explaining the purpose of the interview and interview process to participants, emphasising voluntary participation and confidentiality. Interviews were transcribed and saved in passwordprotected files, with transcripts sent to interviewees to affirm them as true records.

\section{Analysis}

As the aim was to explore and understand perceptions and experiences of TBCA from a wide range of stakeholders, thematic qualitative analysis was considered the most appropriate means by which to identify areas of commonality and difference across the data. In particular, the framework approach to thematic analysis-a systematic, matrix-based method to classify and organise qualitative data-was chosen as the specific thematic approach. ${ }^{28}$ The transparency of framework's analytical method and its step-by-step approach was considered most suitable to an interdisciplinary team and to a policy-oriented study hoping to inform practice. Two researchers (SW and $\mathrm{KJ}$ ) double coded six initial transcripts. Data were analysed noting experiences participants underwent, processes they described, understandings and perceptions they held, motivations they expressed. SW and KJ discussed initial coded sections, proposing their own interpretations and potential categories which would inform the research question. Categories of these common experiences were developed to build an analytical framework (coding index), against which the remaining transcripts were coded. Each main category was then charted within a matrix of rows (individual cases) and columns (codes) with 'cells' of summarised data providing a structure into which the data were systematically reduced. From reading the 
matrices, SW, KJ and SBS discussed emergent concepts and critically explored interviewee responses, comparing their own interpretations. This process was informed throughout by the overall research aim and shaped inductively by ideas that emerged from the data, and deductively from literature reviewed. During this process, the researchers moved from description to interpretation and more explanatory analysis enabling final themes (eg, 'looking ahead', concerning participants' feelings concerning the future of TBCA) to be agreed. An example of the analysis process is provided in the appendix.

\section{RESULTS}

Twenty-six interviews were conducted between 6 January and 14 March 2014 (see table 1) after 1515 TB cases had been reviewed.

\section{Preconceptions}

There were a number of preconceptions about TBCA ranging from concerns about time for form filling and anxiety about scrutiny to optimism about its potential.

\section{Engagement with TBCA}

The TB nurse specialists interviewed had attended all or most of the audits (this is expected as it is TB nurses who present cases). By contrast, half of the consultant physicians had attended half of the audits and half had attended one audit only. Public health practitioners' attendance varied, with consultants in disease control attending most or all audits in their region and epidemiologists/analysts attending fewer audits.

\section{Reasons for attendance/non-attendance}

Part-time TB nurse specialists had problems finding time for both preparation and actual attendance. Some TB nurse specialists felt that managers did not understand the importance of TBCA. Consultants cited time as an issue in their attendance more often than nurse specialists and emphasised the importance of knowing dates in advance. Public health practitioners had the fewest problems in attending as their diaries were less constrained by clinical commitments.
Changes in perspective on TBCA after attendance

Initial fears about preparation time, presenting and scrutiny of practice were not realised. Those who had initially felt positive about the idea of audit actually found it surpassed their expectations. Those who had seemed unsure about the content and purpose of cohort audit before attendance felt it was a useful process.

\section{Presenting cases}

Early TBCA meetings were perceived as more formal and a change towards a more relaxed and supportive atmosphere was noted over time. TB nurse specialists who worked in areas of high prevalence were occasionally challenged by the number of cases they had to present, but with practice, the presentation of simple cases had become routine. For complicated cases, however, TB nurse specialists found it more difficult deciding what to include or leave out of their presentations. The Chair's role was seen as crucial in maintaining pace and pertinence. The most useful aspect of meetings was perceived to be the discussion arising from cases. Discussion was perceived to take place less often when fewer consultant physicians attended.

While public health practitioners said TBCA was important, some expressed mixed feelings about public health participation in meetings, with some expressing ambivalence about the clinical focus.

\section{Buy in from colleagues}

Attendance by consultant physicians was seen as important in changing and improving practice: without their participation, TB nurse specialists felt unsupported. Both TB nurse specialists and consultant physicians felt that public health attendance at TBCA was important. Public health practitioners who did participate stated that they were still working on strategies to encourage more colleagues to participate in TBCA meetings.

\section{Changes attributed to NW TBCA}

Perception of personal benefits and costs of attending TBCA

TB nurse specialists felt TBCA led to a wider understanding and appreciation of their role. This, in turn, has increased motivation to improve outcomes. TBCA was described as providing support, reassurance and a

\begin{tabular}{|c|c|c|c|c|c|c|c|}
\hline \multirow[b]{2}{*}{ Group } & \multirow[b]{2}{*}{ Profession } & \multicolumn{4}{|c|}{ Geographical footprint of place of work } & \multirow[b]{2}{*}{ Total } & \multirow{2}{*}{$\begin{array}{l}\text { Gender ratio } \\
\text { M:F }\end{array}$} \\
\hline & & $\begin{array}{l}\text { North } \\
\text { Manchester }\end{array}$ & $\begin{array}{l}\text { South } \\
\text { Manchester }\end{array}$ & $\begin{array}{l}\text { Cumbria and } \\
\text { Lancashire }\end{array}$ & $\begin{array}{l}\text { Cheshire and } \\
\text { Merseyside }\end{array}$ & & \\
\hline A & TB nurse specialists & 4 & 4 & 3 & 4 & 15 & $2: 13$ \\
\hline B & Consultant physicians & 2 & 2 & 1 & 2 & 7 & $6: 1$ \\
\hline C & Public health practitioners & 1 & 1 & 1 & 1 & 4 & $4: 0$ \\
\hline D & Key informants & 2 & 2 & 2 & 2 & 8 & $6: 2$ \\
\hline
\end{tabular}

Responses were grouped into four main themes: preconceptions, engagement with TBCA, changes attributed to NW TBCA and looking ahead. Illustrative quotes for the key themes are presented in table 2.

F, female; M, male; NW, North West; TBCA, tuberculosis cohort audit. 
Table 2 Key themes, findings interpreted and illustrative quotes

\begin{tabular}{ll}
\hline Themes & Findings \\
\hline Preconceptions & $\begin{array}{l}\text { Views varied widely. Negative views } \\
\text { were mainly concerns about time and } \\
\text { scrutiny of practice (relating to } \\
\text { individuals). }\end{array}$ \\
& \\
& $\begin{array}{l}\text { Positive views expressed optimism } \\
\text { about the potential to improve practice } \\
\text { and outcomes. }\end{array}$
\end{tabular}

Engagement with Participants listed a number of reasons TBCA for their attendance/non-attendance at TBCA. Participants found that attendance brought them a sense of community and enlarged their sphere of influence and support.

There were changes in participants perspective on TBCA after attendance, mainly due to a reassessment of the value of attendance; many expressed that their fears prior to attendance were unjustified.

Participants conveyed a range of experiences of attending audit and presenting cases. Over time people became more confident and relaxed. Consultants felt engaged when their patients were presented.

When discussing whether participants felt they had buy in to TBCA from colleagues.

\section{Illustrative quotes}

"Whenever you hear about things like that, you think 'Oh for goodness sake,' you know 'what more do they want us to do?"' (ID8 TB nurse specialist)

"My concern was getting Trusts to accept it as a valuable or valid part of our work." (ID21 TB nurse specialist)

"I couldn't imagine how that's going to be useful or have an impact until I attended." (ID7 Consultant physician)

"[l thought] it was a perfect change agent for us." (ID10 TB nurse specialist)

"I was very pleased to see that there [would be a] multi-disciplinary forum." (ID25 Public health practitioner)

"It's just become part of practice now, and I do appreciate it very much, what it offers." (ID9 TB nurse specialist)

"It wasn't a witch hunt [the audit process]; it's about trying to get the outcome at the end of it." (ID6 TB nurse specialist) "People say "Well, I did this," or "I did that," and so you think, 'Oh, I might try that next time'." (ID29 TB nurse specialist) "Sometimes you feel a little bit isolated and I think this helps with the isolation." (ID25 TB nurse specialist)

"You have to make your job pay [...] that's the difficult choice of whether you go to cohort review, which earns nothing-nothing [of] monetary value-but a heck of a lot in other ways." (ID21

TB nurse specialist)

"I have always thought they were beneficial and I still think they are beneficial; perhaps at the beginning I didn't appreciate from a learning point of view how much." (ID5 TB nurse specialist) "I was looking forward to the next one from a point of view of you know professional practice, it's very stimulating." (ID11 Public health practitioner)

"It was refreshing just to go through patients from all over the region and see the TB nurses presenting their patients, how much work they put in to achieving good outcomes." (ID34 male, Consultant physician)

"Sometimes it becomes a little bit tiring and stressful when you don't have anything to highlight during them." (ID7 Consultant physician)

"When they are going through the case you get more of an appreciation of what is actually going on and all these things obviously help when it comes to our outbreak support." (ID2 male, Public health practitioner)

"No matter how much you know it's not about judging you personally, when you first start to come that is how it feels and there are still times that you would sit there or a colleague would say "I don't want to present this one l'm not happy with it," and but, you just get on, you grit your teeth, and actually when that's happened to me-I felt supported at the end of it." (ID19 TB nurse specialist)

"We are all a bit more relaxed in presenting our cases." (ID5 TB nurse specialist)

"The more you go the less threatening it becomes." (ID21 TB nurse specialist)

"I've suggested my junior trainees and registrars come, this could help in their career progression." (ID27 Consultant physician)

"If you're going on your own and you've got nobody else there with you, supporting you, it's like; you [consultant physicians] don't really care what we do." (ID6 TB nurse specialist) 
Table 2 Continued

Themes Findings Illustrative quotes

"Our Consultant says that TB comprises of about $5 \%$ of his total workload, so in the scheme of things it's not a huge priority, you know he's sort of balancing it with obviously his cancer patients and bronchoscopy lists, everything else, so I don't really feel he intends to come." (ID23 nurse specialist)

"I think they do need to have quite a good input into public health, because obviously TB you know it has implications on public health and especially if there's been sort of an outbreak situation." (ID12 TB nurse specialist)

Changes attributed to NW TBCA
Personal benefits and costs to individuals of attending TBCA related to communication community and learning.
Individuals perceived a range of benefits and costs of TBCA to service/ footprint. Some felt empowered to use the TBCA outcomes and data to make service changes. TBCA was seen to reduce risk to patients and practitioners, act as a source of support and advice when cases are complicated (especially in low incidence areas and for lone workers).

As mentioned in previous findings, participants described an overall picture of a new community of practice which developed through attendance at TBCA.
"It makes me think about what I am doing, the care I am giving, it makes me sure I tick those boxes but I also know why I am ticking them boxes and why it is important." (ID5 TB nurse specialist)

"It does make people a bit more aware of the extent of the difficulty of doing our work." (ID8 TB nurse specialist)

"It's good to talk about your ideas with some of the Consultants that you don't work with directly." (ID14 TB nurse specialist) "You think your patients are all doing well then you go to a TB cohort review and [see that] actually $25 \%$ of patients with TB in this area died, and you think 'ah...I didn't... really appreciate that, I've seen a couple die, but I didn't know...the big picture." (ID34 Consultant physician)

"Now it's my own patients it's much more interesting, and actually getting feedback on the things that I should have done or shouldn't have done, is even more helpful. I love it, they are very interesting." (ID32 Consultant physician)

"It's been good to help push the Trust in the way that TB patients should be cared for-and how the service should be managed, l've been able to use it as leverage to say 'this is what's needed...because you're being watched now." (ID21 male TB nurse specialist)

"If something's presented at cohort which is not meeting an adequate standard then intervention will occur. It got picked up because of audit, it got highlighted because of audit, and action took place because of audit." (ID19 female TB nurse specialist) "You can raise issues and challenge, in a positive way, management of patients in different areas. The only problem is that the people who really need to be listening are not coming." (ID34 Consultant physician)

"The worst thing in the world is for there to be a disconnect between policy-makers, strategists, public health and clinical services, that's disastrous." (ID13 Public health practitioner) "The way that funding is obtained for TB control now is, has to be kind of negotiated locally now, so we need this information to feedback to Commissioners so that they understand the burden of disease and the particular issues the need for perhaps additional workforce." (ID26 Public health practitioner) "It's sort of built this idea of collegiate working, you know that you want to work together, that you are colleagues and you can help each other out." (ID19 TB nurse specialist)

"It just builds bridges really, it's a lot easier free-flowing information and they'll help us, and we'll help them." (ID10 TB nurse specialist)

"I think it's developed into much more than just data collection, it's ensuring that the patients are getting what the patient deserves, getting the treatment that is the right treatment, in a timely manner, with the best outcomes." (ID1 TB nurse specialist) 
Table 2 Continued

\begin{tabular}{ll}
\hline Themes & Findings \\
\hline & Individuals listed numerous changes of \\
practice they attributed to attendance & at TBCA, from their individual practice \\
to service changes, including a \\
renewed sense of co-working to \\
improve outcomes and an appreciation \\
of the bigger picture and complexity of \\
TB care and control in the region.
\end{tabular}

\section{Illustrative quotes}

"It's also about reminding people for the best investigations, for example thoracoscopy for pleural TB rather than just pleural aspirations so some of the improvement is documented and some of it isn't." (ID32 Consultant physician) "For the patients it means that their treatment has been gone through a recognised panel of people or a cohort of a panel which is quality assurance really." (ID3 Consultant physician) "It's sort of a reflective learning exercise for us and the TB nurse specialists to see what could have been done better or what could have been done differently." (ID7 Consultant physician) "There's a collective sort of ownership that, we as a group of people involved in TB control, public health, TB services (agree targets) and so they're meeting their own targets that we've all set, they're not meeting something that I, from outside have imposed on them, or even that their bosses have imposed on them." (ID13 Public health practitioner)

Looking ahead When they were asked how they would feel if TBCA did not continue, participants uniformly did not want to see that happen and felt it could be a risk to patients, service delivery and TB care in the NW.

Key informants

Key informants had an in-depth understanding of the inception and function of TBCA in the NW.
"TB is a condition that can't just be looked at locally, it has to be looked at on a bigger picture, regionally, nationally and internationally, and they all feed into each other, and they're all interdependent of each other, and so to go back to just being in that one little pot you lose so much, we can't put a fence around [our area] and say 'that's us done, on your way." (ID19 TB nurse specialist)

"[How would you feel if TBCA stopped?] 'Gutted really'." (ID5 TB nurse specialist)

"I feel like it's a safety net." (ID25 TB nurse specialist)

"I think if the cohort review wasn't there, there is the possibility that it would slip and other things take over and patients slip through the net, 'cause it's human nature, it will happen; I think cohort review keeps us on track and I think it really has a direct impact on patient safety." (ID10 TB nurse specialist) "I think it would be a disaster." (ID7 Consultant physician) "The consequences of getting TB care wrong are so high-even in costs terms alone-not to mention the impact on the patient of course, but the cost of treating someone for MDR TB for example are so much higher than a case of standard TB that we just can't afford not to do things properly, and if the cohort review is what it takes then l'm sure that's a highly cost effective way of preventing even a single case of MDR TB in a year, and that, you know that would more than pay for the costs of the meeting and the administration and everything." (ID32

Consultant physician)

"If cohort review becomes part and parcel and owned by, if you like, the local health economy, that ownership I think makes it possibly more likely that it will feed into commissioning." (ID13 Public health practitioner)

'You're saving lives, you can really see that you've influenced practice; it's incredible to see. I just think it's really powerful, it's really rare in public health to see such improvement so quickly." (ID31 Key informant)

There have been specific instances where it's made a radical change to the way a patient's been treated. And then there's been a more collective changes, [for example] if you look at treatment completion rates." (ID24 key informant) "The biggest failing is that we haven't been able to do the reporting structure." (ID31 key informant) 
place to ask questions. This was especially important for new members of staff, particularly TB nurse specialists working on their own (some TB nurse specialists working in the community did not regularly meet with consultant physicians). TB nurse specialists identified opportunities for reflective practice and regular ringfenced time together with peers as particular benefits.

Consultants enjoyed receiving feedback on their practice and felt more involved with TBCA when their own patients were presented. Most consultants in areas of low prevalence recognised the importance of TBCA in assessing their current practice. One doctor felt frustrated that TBCA does not provide an arena for more in-depth discussion which could improve the service, even though TBCA was seen as educational and providing the 'bigger picture'.

Both TB nurse specialists and consultant physicians felt grateful for support from senior colleagues at TBCA (including the chair) with complex cases or queries about practice, and were reassured that the chair of the TBCA would address any issues that were potentially putting patients at risk.

Public health practitioners felt that attendance at TBCA provided them with a stronger link to clinical practice than existed previously as well as deeper intelligence about local epidemiology.

\section{Perceived benefits and costs of TB audit to service delivery}

TBCA was thought by all groups to provide learning about practices in areas with differing resource availability and patient demographics which affect complexity and contact screening decisions. This cross-learning also helped in using TBCA as evidence for change with management at service or Trust level.

\section{Community}

All the groups interviewed felt that the "community of practice' for TB in the NW had changed. Greater contact with the wider team created mutual respect between different roles. Individuals felt more able to ask for help or to collaborate between catchment areas to ensure better patient care. Public health practitioners explained that previous attempts to link public health with TB teams had not been successful.

\section{Changes in practice}

Several service changes were identified as a result of TBCA targets including increased HIV testing and increased speed of notification to ETS.

TB nurse specialists felt that TBCA empowered them to question consultant physicians after attending TBCA and found TBCA provided evidence to their managers of their performance. They had all changed documentation procedures, describing the use of the TBCA form as a checklist to ensure everything from diagnosis to discharge is managed correctly. There was a perception of increased contact-tracing activity.
Consultants generally described fewer changes to practice than TB nurse specialists and viewed TBCA as a promising forum for quality improvement.

Public health practitioners were less willing to attribute changes in practice to TBCA until more evaluation has been done but did describe TBCA as creating more interest and a higher profile for TB care. They noted that data quality and timeliness of reporting has improved markedly as a result of TB nurse specialists' effort and commitment. Like the consultant physicians, they questioned whether lessons learnt were being adequately collated and disseminated and thought data from TBCA will be a rich source for research and national intelligence.

TBCA was not seen by any group as a 'tick box' or data collection exercise.

\section{Looking ahead}

All groups of participants expressed a desire for TBCA to continue and felt heavily invested in the process.

When asked how they would feel if TBCA stopped, TB nurse specialists said it would be a missed opportunity. The biggest impact was thought to be on new and isolated staff. Failure to continue with TBCA in the NW was seen as a short sighted and backwards step which would probably reduce patient safety and quality of care.

Consultants noted it was 'still early days' and that TBCA was only really now getting properly established. Although too soon to evaluate the difference it was making, it was considered that loss of TBCA would be detrimental to the NW and may result in more mistakes being made.

Public health practitioners thought that more evaluation was needed to be done before the impact of TBCA could be assessed. They felt that keeping a NW structure is important but that there needed to be a mechanism to feed data into wider service improvement and public health processes.

\section{Key informants}

Themes emerging from the eight interviews with key informants were compared with the results from the three main respondent groups as a method of validation. The key informants differed from the main respondents in that they had more in-depth understanding of the context and process of TBCA because of their participation in the TBCA Steering Group and/or their experience of chairing TBCA meetings.

Key informants corroborated the views of other groups. There was optimism about the potential of TBCA to improve care in the NW, especially in areas of lower incidence where it was felt care may be less effective. There was concern about the practicalities and about the extent to which consultant physicians would continue to engage in the process.

Key informants described the setting up of TBCA as a systematic and strategic process that involved the establishment of a steering group, a detailed engagement 
strategy and appointment of a dedicated coordinator; these were felt to be critical to the perceived success of the NW TBCA.

TBCA was thought to be a good way to look at workforce generally across a footprint and to understand the difficulties facing a diverse workforce. The geographical isolation of some TB nurse specialists in the NW made TBCA more relevant and important.

TBCA was described as an innovative system similar to the new field of sector-led improvement ${ }^{29}$ and that it is the increased sense of community of practice which has been the most surprising and potentially the most important improvement from TBCA.

Realising the true potential of TBCA in the NW will be dependent on securing funding into the long term. Participants strongly suggested that if TBCA is discontinued, it would be difficult or impossible to start up again and that serious financial and patient risk issues could result.

\section{Community of practice}

Running through the participant narratives were recurring themes which describe the inception of a 'community of practice'. This concept was constructed from depictions of a renewed engagement as a collective to collaborate and engage in regional TB control. The mainly negative preconceptions of TBCA were unsubstantiated and attendance at TBCA built confidence and trust in the process. Participants gained personal benefit from attending TBCA which was due to a sense of support, learning, communication and coming together.

Engagement with the process and perceived benefits and costs varied by professional group. Each group also had differing reasons for attendance or non-attendance and suggestions for improvements to the process.

Participants were unanimous that if TBCA ceased to function, this would be a loss to them and would damage TB care in the NW.

\section{DISCUSSION}

Our study is the first to evaluate the implementation of TBCA using qualitative methods to explore attendees' perceptions of the impact of TBCA and also the first to evaluate implementation of TBCA over a large geographic area in the UK.

As demonstrated by the results, the key finding was a description of collaborative working and the development of a community of practice with a common purpose. ${ }^{30}$ TB services across the UK have reported weaknesses in communication between sectors with fewer than half of TB services having local arrangements to work with local authorities and social care services. ${ }^{31}$ Lack of communication and collaboration in healthcare teams has negative effects on patient outcomes with failure of communication being the primary cause for the majority of errors, ${ }^{32}$ and outbreaks of TB have been shown to frequently originate from clinical mistakes in diagnosing and treating TB. ${ }^{33}$ Reducing the burden of $\mathrm{TB}$ in the UK requires the coordinated action of many partners, working together across local authority and NHS boundaries. ${ }^{5}$

Communities of practice have been described as 'groups of people who share a concern, a set of problems or a passion about a topic, and who deepen their knowledge and expertise in this area by interacting on an ongoing basis'. ${ }^{44}$ They have been found to break down professional, geographical and organisational barriers, reduce professional isolation and facilitate the implementation of new processes and technology. Significant improvements in the delivery of healthcare and patient experience have been found when clinicians work across the patient pathway and span organisational boundaries. ${ }^{35}$ Therefore, the demonstration of an effective and robust community of practice originating from attendance at the NW TBCA is a significant finding.

TB nurse specialists in this study had a wide variety of working patterns and many were practising on their own in low incidence rural areas. Nonetheless, they described how they felt increased acknowledgement of their practice, and they experienced support and reassurance which improved their morale. TBCA was felt to be especially important for those nurses who did not work in a TB team, both to raise morale and improve standards. Empowerment as a result of closer linkage to colleagues both across footprints and between disciplines was described in this study and has previously been shown to improve job satisfaction and patient outcomes. ${ }^{36}$ Since the completion of this analysis, TB nurse specialists have been paired with clinical chairs and this is likely to lead to enhanced leadership skills which can contribute to a reduction in power differentials and improve interprofessional collaboration. ${ }^{37}$

Clinicians saw TBCA primarily as a way of preventing mistakes and improving individual practice. Contemporary data looking at avoidable harm demonstrate that NHS staff do not speak out often enough and that this can be rectified. ${ }^{38}$

Public health practitioners were able to attend TBCA but some found the clinical focus off-putting (although this contradicted the value they placed on TBCA as a link to clinical practice). This may have led to some disengagement. ${ }^{39}$ Successful involvement of multiple professional groups requires understanding of clear shared purpose and processes, ${ }^{40}$ and this will require more contribution from the public health perspective. ${ }^{41}$

Participant experiences of TBCA made it clear that the voices and opinions of all attendees, irrespective of role or experience, are actively encouraged and valued which is one component of successful improvement collaboratives. ${ }^{42}$

Interviews with key informants described meticulous planning by the steering group which ensured key players were involved from the beginning, and many individuals within the participant groups expressed they had subsequently encouraged colleagues to attend, 
taking it on themselves to champion change. Other participants described a process of engagement which was facilitated by encouragement from these 'local change champions' whose importance has been cited elsewhere. ${ }^{43}$ The emphasis by TBCA leaders (Chairs and Steering Group) on inclusiveness may have been a key factor in promoting the 'psychological safety' of participants in cross-disciplinary engagement. ${ }^{44}$

Weaknesses within TBCA described by study participants included the lack of planned ongoing funding for what needs to be a long-term process. At the time of data analysis, the lack of a report for outside dissemination on the TBCA's outcomes was seen as a failing by participants, affecting ongoing issues with engagement and funding. A report on outcomes has since been published. ${ }^{17}$ The delay in producing a report was due to lack of infrastructure and funding to provide technical assistance and analysis to TBCA. Initiatives like TBCA that span geographical and disciplinary boundaries need background resources and structure to facilitate and embed improved integration of services. ${ }^{45}$

Although the effectiveness of TBCA had previously been evaluated using quantitative methods analysing clinical outcomes of TB treatment, no studies have assessed contextual factors in the successful implementation (defined as being made workable and integrated in everyday healthcare practice ${ }^{46}$ ) of TBCA or evaluated participant experience. The use of in-depth one-to-one interviews allowed us to explore a complex process by examining the perceptions and shared experiences of TBCA. ${ }^{47}$

Our data set included a large number of stakeholders from the three main cadres who participate in TBCA, across four geographic footprints, purposely sampled to attain a wide range of disciplines and working practices. Data analysis found recurrent themes that indicated saturation, ${ }^{48}$ especially within the $\mathrm{TB}$ nurse specialist profession.

Triangulation through observation at TBCA and interviews with key informants were used to ensure a deeper understanding of the experience and process of TBCA, to feed into interview topic guides, inform analysis of participant interviews and elicit data relating to the implementation of the NW TBCA. The themes evolving from framework analysis of the data were further validated $^{49}$ by the use of two researchers to double code the transcripts and discussion with a further author on emergent themes.

Limitations of this study are the comparatively smaller data set from the consultant physician and public health practitioner professions which means that there may be important perceptions of TBCA within these groups that were missed.

Information on participant preconceptions of TBCA were only collected after participants had experienced cohort review which could introduce recall bias.

The unique geographic configuration and implementation of the NW TBCA may mean that the findings are not easily transferred to other areas without careful adaptation.

Our findings support those of the report on staff experience of the NCL TB Service TBCA in 2010 from an online questionnaire. ${ }^{11}$ In agreement with our findings, staff at NCL felt TBCA identified gaps in services and training needs, and a majority felt their working practice had changed due to attendance at TBCA (mainly through an increased focus on contact tracing and improved documentation).

Earlier studies suggest that unidisciplinary communities of practice retard the spread of innovation ${ }^{50}$; it may be that the TBCA differs by allowing knowledge transport to be facilitated between professions by the use of chairs as 'brokers'. ${ }^{51}$

TBCA appears to provide a mechanism for staff to speak up about unsafe practice and organisational issues that affect care. Further, it appears that TBCA contributes to more interaction between historically unequal professions which could make self-directed culture change possible. ${ }^{52}$

\section{Implications of our findings}

The fact that TBCA resulted in collaborative working and the development of a community of practice with a common purpose is a significant finding and suggests that the NW model of TBCA can be an important means of quality improvement in similar contexts. ${ }^{53}$ PHE's new TB Strategy ${ }^{5}$ promotes a coordinated approach to $\mathrm{TB}$, involving NHS commissioners, providers, local government and PHE through TB Control Boards. The model of TBCA described here provides an important coordination mechanism through which the 'soft data' of process and learning can be an explicit part of the TB Strategy. The benefits of communities of practice tend to accrue over time. The results of this study will be valuable to the TBCA Steering Committee for reflection on how to further strengthen the community of practice and a follow-up, qualitative process evaluation could be valuable in the future. ${ }^{54}$ In such an evaluation, it will be important to include individuals who choose not to attend TBCA in order to gain insights into the barriers to attendance.

\section{CONCLUSION}

The NW TBCA model has enabled cross-professional collaboration and the establishment of a community of practice which is facilitating a culture of shared experience, open communication and respect. This community of practice is vital as a tool, which should be sustained and further improved for continued quality improvement in TB control. Further qualitative study of practitioners who are resistant to attending TBCA may provide a better understanding of how to facilitate attendance.

The paper demonstrates the operational perspectives which underpin development of a powerful and robust 
multidisciplinary community of practice across a wide geographical area. It will inform the establishment of effective TB cohort audits across the new TB Control Board footprints (as established by PHE's new TB Strategy).

\section{Author affiliations}

${ }^{1}$ Department of Clinical Sciences and Centre for Applied Health Research \& Delivery, Liverpool School of Tropical Medicine, Liverpool, Merseyside, UK ${ }^{2}$ Department of Public Health and Policy, University of Liverpool, Liverpool, Merseyside, UK

${ }^{3}$ Manchester Royal Infirmary, Manchester, UK

${ }^{4}$ Public Health England, Liverpool, UK

${ }^{5}$ Bolton NHS Trust, Bolton, UK

${ }^{6}$ North Manchester General Hospital, Manchester, UK

${ }^{7}$ Liverpool Community Health Trust, Liverpool, UK

${ }^{8}$ Liverpool Heart and Chest Hospital, Liverpool, UK

${ }^{9}$ Royal Liverpool University Hospital, Liverpool, UK

Twitter Follow Selina Wallis at @unlockingbirth and Bertie Squire @BertieSquire

Acknowledgements The authors thank all those who took part in this work and especially those who set aside time to participate in the in-depth interviews.

Contributors The study was conceived and designed by SBS and KD with support from MW, PC, SF, PM, CW and JW. The protocol was finalised by SW who collected all the data. Analysis was conducted by SW and KJ. SW led the writing guided by KJ and SBS, with additional comments from the remaining authors. All authors read and approved the final manuscript.

Funding This research was supported by Public Health England and the Centre for Applied Health Research and Delivery, Liverpool School of Tropical Medicine (LSTM).

Disclaimer The views and opinions expressed are those of the authors and do not necessarily reflect those of Public Health England or LSTM.

Competing interests The following authors were members of the NW TB Cohort Audit Steering Group at the time of the study: MW, PC, KD, SF, PM, CW, JW, DS, SBS. They contributed to the study design and the writing of the paper. However, the interviewing and data analysis were the sole responsibility of SW and KJ who were independent of all Cohort Audit processes.

Ethics approval Liverpool School of Tropical Medicine Research Ethics Committee (ref no 13.37).

Provenance and peer review Not commissioned; externally peer reviewed.

Data sharing statement No additional data are available.

Open Access This is an Open Access article distributed in accordance with the Creative Commons Attribution Non Commercial (CC BY-NC 4.0) license, which permits others to distribute, remix, adapt, build upon this work noncommercially, and license their derivative works on different terms, provided the original work is properly cited and the use is non-commercial. See: http:// creativecommons.org/licenses/by-nc/4.0/

\section{REFERENCES}

1. Care Quality Commission. NHS England (2014). NHS five year forward view. London: NHS England, 16-16. [Online]. Available from: https://www.england.nhs.uk/wp-content/uploads/2014/10/ 5yfv-web.pdf

2. Belay ED, Monroe SS. Low-incidence, high-consequence pathogens. Emerg Infect Dis 2014;20:319.

3. Jereb J. Progressing toward tuberculosis elimination in low-incidence areas of the United States. Morb Mortal Wkly Rep 2002;51:1-16.

4. Lönnroth K, Migliori GB, Abubakar I, et al. Towards tuberculosis elimination: an action framework for low-incidence countries. Eur Respir J 2015;45:928-52.

5. Lipman M, White J. Collaborative tuberculosis strategy for England. BMJ 2015;19;350:h810.
6. van Hest NA, Aldridge RW, de Vries G, et al. Tuberculosis control in big cities and urban risk groups in the European Union: a consensus statement. Euro Surveill 2014;19:pii: 20728.

7. Damschroder LJ, Aron DC, Keith RE, et al. Fostering implementation of health services research findings into practice: a consolidated framework for advancing implementation science. Implement Sci 2009;4:50

8. World Health Organization. Implementing the WHO Stop TB Strategy: a handbook for national TB control programmes. World Health Organization; 2008.

9. Munsiff S, Ahuja S, King L, et al. Ensuring accountability: the contribution of the cohort review method to tuberculosis control in New York City. Int J Tuberc Lung Dis 2006;10:1133-9.

10. National Institute for Health and Clinical Excellence. (2012) Identifying and managing tuberculosis among hard-to-reach groups. [Online]. Available from: https://www.nice.org.uk/guidance/ng33/ evidence/appendix-n-ph37-80851860866 (accessed 11 Mar 2016).

11. Anderson C, White J, Dart S, et al. Evaluation of the implementation of cohort review by North Central London TB Service. Health Protection Agency; 2011. [Online]. Available from: http://www.cmft. nhs.uk/media/440509/3cohort\%20review.pdf

12. Lalor MK, Pedrazzoli D, Davidson JA, et al. Tuberculosis in the UK, 2014 report. [Online]. Available from: https://www.gov.uk/ government/uploads/system/uploads/attachment_data/file/360335/ TB_Annual_report_4_0_300914.pdf

13. Ploubidis GB, Palmer MJ, Blackmore $\mathrm{C}$, et al. Social determinants of tuberculosis in Europe: a prospective ecological study. Eur Respir $J$ 2012;40:925-30.

14. Public Health England. (2015) Tuberculosis in England: 2015 report version 1.1. Public Health England: London. [Online]. Available from: https://www.gov.uk/government/uploads/system/uploads/attachment data/file/492431/TB Annual_Report v2.6 07012016.pdf

15. Hoppe LE, Kettle R, Eisenhut M, et al. Tuberculosis-diagnosis, management, prevention, and control: summary of updated NICE guidance. BMJ 2016;352:h6747.

16. Wake C. North West TB regional audit-cohort review handbook. North West TB Cohort Audit Steering Group, 2013. [Online]. Available from:. https://tbsummit.wordpress.com/2013/03/26/ nw-regional-tb-regional-audit-cohort-review-handbook/

17. North West TB Cohort Audit Steering Group. North West TB cohort audit 2014 report. 2014. 3. [Online]. Available from: https://tbsummit. files.wordpress.com/2013/12/nw-tb-cohort-audit-2014-report-final.pdf

18. MacPherson PS, Stephen Bertel MD BChir MD, et al. Equitable tuberculosis care in the North West of England: analysis of TB cohort review data. Int J Tuberc Lung Dis 2016. In press.

19. Campbell M, Fitzpatrick R, Haines A, et al. Framework for design and evaluation of complex interventions to improve health. BMJ 2000;321:694-6.

20. Green J, Thorogood N. Qualitative methods for health research. Sage, 2013.

21. King N, Horrocks C. Interviews in qualitative research. Sage, 2010.

22. Bryman A. Social research methods. Oxford University Press, 2012.

23. Guion LA. Triangulation: Establishing the validity of qualitative studies. University of Florida Cooperative Extension Service, Institute of Food and Agricultural Sciences, EDIS; 2002.

24. Tong A, Sainsbury P, Craig J. Consolidated criteria for reporting qualitative research (COREQ): a 32-item checklist for interviews and focus groups. Int J Qual Health Care 2007;19:349-57.

25. Patton MQ. Qualitative analysis and interpretation. Qual Res Eval Methods 2002:3:431-539.

26. Jefferson L, Bloor K, Maynard A. Women in medicine: historical perspectives and recent trends. Br Med Bull 2015;114:5-15.

27. Sofaer S. Qualitative research methods. Int J Qual Health Care 2002;14:329-36.

28. Gale NK, Heath G, Cameron E, et al. Using the framework method for the analysis of qualitative data in multi-disciplinary health research. BMC Med Res Methodol 2013;13:117.

29. Holmes D, Brookes C. Sector-led improvement in children's services: a lever for evidence-informed practice? Evid Policy 2014;10:513-25.

30. The Meaning of a Healthcare Community of Practice. Nursing forum Wiley Online Library, 2015.

31. Abubakar I, Lipman M, Anderson C, et al. Tuberculosis in the UKtime to regain control. BMJ 2011;343:d4281.

32. Arnaudova A. Eighth futures forum: on governance of patient safety. World Health Organization, 2005

33. van der Werf MJ, Blasi F, Giesecke J, et al. Lessons learnt in Europe on tuberculosis surveillance, outbreaks and BCG vaccination in 2011. Eur Respir J 2013;41:767-71. 
34. Wenger E, McDermott RA, Snyder W. Cultivating communities of practice: a guide to managing knowledge. Harvard Business Press, 2002.

35. Woodard F, Weller G. An action research study of clinical leadership, engagement and team effectiveness in working across NHS boundaries. Work Based Learn eJ 2011;1:80-119.

36. Hughes R. Nurses at the "Sharp End" of Patient Care. In: Hughes RG, editor. Patient Safety and Quality: An Evidence-Based Handbook for Nurses. Rockville (MD): Agency for Healthcare Research and Quality (US); 2008 Apr. Chapter 2. [Online]. Available from: http://www.ncbi.nlm.nih.gov/books/NBK2672/

37. Bell $\mathrm{AV}$, Michalec $\mathrm{B}$, Arenson $\mathrm{C}$. The (stalled) progress of interprofessional collaboration: the role of gender. $J$ Interprof Care 2014;28:98-102.

38. Reid J. Speaking up: a professional imperative. J Perioper Pract 2013;23:114-18.

39. Martin-Misener R, Valaitis R, Wong ST, et al, Strengthening Primary Health Care through Public Health and Primary Care Collaborations Team. A scoping literature review of collaboration between primary care and public health. Prim Health Care Res Dev 2012;13:327-46.

40. Raine R, Xanthopoulou P, Wallace I, et al. Determinants of treatment plan implementation in multidisciplinary team meetings for patients with chronic diseases: a mixed-methods study. BMJ Qual Saf 2014;23:867-76.

41. Santana C, Curry LA, Nembhard IM, et al. Behaviors of successful interdisciplinary hospital quality improvement teams. J Hosp Med 2011;6:501-6.

42. Hulscher ME, Schouten LM, Grol RP, et al. Determinants of success of quality improvement collaboratives: what does the literature show? BMJ Qual Saf 2013;22:19-31.

43. Kirchner JE, Parker LE, Bonner LM, et al. Roles of managers, frontline staff and local champions, in implementing quality improvement: stakeholders' perspectives. J Eval Clin Pract 2012;18:63-9.
44. Nembhard IM, Edmondson AC. Making it safe: the effects of leader inclusiveness and professional status on psychological safety and improvement efforts in health care teams. J Organ Behav 2006;27:941-66.

45. Nasir L, Robert G, Fischer M, et al. Facilitating knowledge exchange between health-care sectors, organisations and professions: a longitudinal mixed-methods study of boundary-spanning processes and their impact on health-care quality. Southampton (UK): NIHR Journals Library; 2013 Oct. (Health Services and Delivery Research, No. 1.7.) http://www.ncbi.nlm.nih.gov/books/NBK262946/ doi:10. 3310/hsdr01070

46. May C, Finch T, Mair F, et al. Understanding the implementation of complex interventions in health care: the normalization process model. BMC Health Serv Res 2007;7:148.

47. Dicicco-Bloom B, Crabtree BF. The qualitative research interview. Med Educ 2006;40:314-21

48. Ritchie J, Lewis J, Elam G. Designing and selecting samples. Qual Res Pract 2003;2:111-45.

49. Jonsen $\mathrm{K}$, Jehn KA. Using triangulation to validate themes in qualitative studies. Qual Res Organ Manag 2009;4:123-50.

50. Ferlie E, Fitzgerald L, Wood M, et al. The nonspread of innovations: the mediating role of professionals. Acad Manage $J$ 2005;48:117-34.

51. Tasselli S. Social networks and inter-professional knowledge transfer: the case of healthcare professionals. Organ Stud 2015;36:841-72.

52. Davies HT, Mannion R. Will prescriptions for cultural change improve the NHS? BMJ 2013;346:f1305.

53. Urquhart R, Cornelissen E, Lal S, et al. A community of practice for knowledge translation trainees: an innovative approach for learning and collaboration. J Contin Educ Health Prof 2013;33:274-81.

54. Wenger E, Trayner B, de Laat M. Promoting and assessing value creation in communities and networks: a conceptual framework. The Netherlands: Ruud de Moor Centrum, 2011. 